\title{
Hilft Nicotinamid?
}

\section{Hier steht eine Anzeige.}

Fragestellung: Hat Nicotinamid bei Patienten mit Friedreichscher Ataxie einen Springer

Hintergrund: Die Friedreichsche Ataxie ist die häufigste erbliche Ataxie mit einer Prävalenz von 1 zu 40.000 Menschen. Aufgrund eines Gendefekts kommt es zu einer geringeren Produktion des Frataxinproteins. Bei transgenen Mäusen zeigte sich, das Nicotinamid (Vitamin B3) die Expression von Frataxin positiv beeinflussen kann.

Patienten und Methodik: Die in Großbritannien durchgeführte, exploratorische offene Dosisfindungsstudie war primär eine Sicherheitsstudie und sollte das Behandlungskonzept überprüfen. Zehn Patienten mit Friedreichscher Ataxie im Alter über 18 Jahren erhielten zunächst Einzeldosen von Nicotinamid. Im zweiten Teil der Studie erhielten acht Patienten über fünf Tage aufsteigend 2-8g Nicotinamid oral. In der dritten Phase erhielten alle zehn Patienten über acht Wochen Nicotinamid. Der primäre Zielparameter war die Expression von Frataxin. Außerdem wurden Sicherheit, Verträglichkeit und die mögliche klinische Wirkung untersucht. In Phase 1 und 2 wurden die Frataxin-mRNA-Expression und die Frataxinproteinkonzentration vor sowie zwei, vier und acht Stunden nach der Gabe von Nicotinamid gemessen. Die klinischen Untersuchungen benutzten die Scale for the assessment and rating of ataxia (SARA) und die Spinocerebellar ataxia functional index (SCAFI).

Ergebnisse: Die Patienten waren im Mittel 33 Jahre alt, die Krankheit hatte im Schnitt mit 20 Jahren begonnen. Nicotinamid in hohen Dosierungen wurde gut vertragen, die einzige Nebenwirkung war Übelkeit. In allen drei Studienphasen zeigte sich eine signifikante Hochregulation der Frataxinexpression mit einer Reduktion der Heterochromafinmodifikation am FXN-Locus. Erwartungsgemäß zeigten die klinischen

Libri V, Yandim C, Athanasopoulos $S$ et al. Epigenetic and neurological effects and safety of high-dose nicotinamide in patients with Friedreich's ataxia: an exploratory, open-label, dose-escalation study. Lancet 2014; 384: $504-13$

\section{Skalen keine Veränderungen.}

Schlussfolgerungen: Hohe Dosen von Nicotinamid führten zu einer Hochregulation der Frataxinkonzentrationen mit entsprechenden Veränderungen der Genexpression.

\section{- Kommentar von Hans-Christoph Diener, Essen}

\section{Diese kleine Studie könnte bedeutsam sein}

Diese kleine Studie könnte von großer Bedeutung für die Behandlung der Friedreichschen Ataxie sein. Die Studie zeigte eine dosisabhängige Hochregulation des Frataxinproteins bei Friedreich-Patienten unter einer Behandlung mit Nicotinamid. Die Behandlung wurde gut vertragen. Erwartungsgemäß ist bei einer kleinen Studiengruppe und kurzer Behandlungszeit kein Einfluss auf die klinischen Symptome der Friedreichschen Ataxie zu erwarten. Erwähnenswert ist, dass die eingeschlossenen Patienten bereits lange erkrankt waren und damit auch keine rasche Besserung der Symptome zu erwarten war. Es sollten jetzt größere Phase-II-Studien bevorzugt an jüngeren Friedreich-Patienten durchgeführt werden. Dann wird sich zeigen, ob eine Langzeitbehandlung mit Nicotinamid entweder das Fortschreiten der Erkrankung verlangsamt oder sogar zu einer Besserung der Symptome führt. 